\title{
Relação entre o craving por tabaco e o craving por crack em pacientes internados para desintoxicação
}

Relationship between craving for tobacco and craving for crack in patients hospitalized for detoxification

Taís Cardoso de Zeni', Renata Brasil Araujo²

\section{RESUMO}

Objetivo: Verificar se há relação entre aumento do craving por crack e aumento do craving por tabaco em pacientes internados para desintoxicação. Método: Ensaio clínico tipo quase-experimental de análise quantitativa. Amostra composta por 32 homens dependentes de cocaína (crack) e tabaco, em duas a três semanas de abstinência. Realizou-se intervenção em grupo, no qual, inicialmente, foram aplicados: CCQB (Cocaine Craving Questionnaire-Brief), QSUB (Questionnaire of Smoking Urges-Brief) e BAI (Inventário Beck de Ansiedade). Em seguida, foram aplicadas imagens relacionadas ao crack e reaplicados CCQB, QSUB e BAI. Após, foi realizada entrevista individual em que se aplicaram FSD (Ficha com Dados Sociodemográficos) e FTND (Fagerström Test for Nicotine Dependence). Resultados: A partir da exposição de imagens relativas ao crack, houve aumento significativo do craving por crack, do craving por tabaco e dos sintomas de ansiedade, estando essas medidas correlacionadas positivamente entre si. Conclusão: Os resultados indicam uma associação significativa entre craving por crack e craving por tabaco, sugerindo que a abstinência de tabaco pode ajudar na eficácia do tratamento para dependência de crack.

\section{ABSTRACT}

Objective: Verify if there is a relationship between crack craving increase and tobacco craving increase in patients hospitalized for detoxification. Method: Quasi-experimental clinical trial using a quantitative analysis. Sample comprised 32 crack-cocaine and tobacco-dependent males, in 2 to 3 weeks of abstinence. A group intervention was conducted to which initially Cocaine Craving Questionnaire-Brief (CCQB), QSUB (Questionnaire of Smoking Urges-Brief) and BAI (Beck Anxiety Inventory) were applied. Next, crack related images were applied and CCQB, QSUB and BAI were reapplied. After that, an individual interview was conducted in which a form containing sociodemographic information and data related to the pattern of consumption of psychoactive substances (FSD) and Fagerström Test for Nicotine Dependence (FTND) were applied. Results: Following the crack related images exposure, there was a significant increase of crack craving, tobacco craving

\section{Keywords}

Cocaine (crack), tobacco, craving, treatment. and anxiety symptoms, being them positively correlated. Conclusion: Results show a significant association between crack craving and tobacco craving, suggesting that tobacco abstinence can help on effectiveness of cocaine dependence treatment.

1 Universidade Federal do Rio Grande do Sul (UFRGS), Escola de Saúde Pública (ESP/RS).

2 Pontifícia Universidade Católica do Rio Grande do Sul (PUCRS), Hospital Psiquiátrico São Pedro (HPSP/RS). 


\section{INTRODUÇÃO}

O craving (ou fissura) pode ser definido como um intenso desejo de utilizar uma específica substância, o qual engloba também, para alguns autores, a intenção de realizar esse desejo, a antecipação dos efeitos reforçadores associados ao uso e o alívio do afeto negativo e dos sintomas relacionados à abstinência ${ }^{1,2}$. O craving deve ser considerado uma variável importante a ser observada no tratamento da dependência química, pois deixa o dependente vulnerável ao abuso de drogas e pode levá-lo à recaída e ao abandono terapêuti$\mathrm{co}$, mesmo diante da vontade convicta de manter-se abstinente e/ou depois de grandes períodos de abstinência ${ }^{1,2}$. No caso específico da dependência de crack, o craving é descrito como incontrolável pelos usuários, levando-os, em sua maioria, ao uso compulsivo, com padrão diário de consumo e por até nove dias contínuos, sendo só finalizado quando é atingido o esgotamento físico, psíquico e/ou financeiro 3 . Diante dessa realidade, o manejo do craving adquire grande importância para a eficácia do tratamento de dependentes de crack.

O craving pode ser desencadeado pelo que se denominam gatilhos - situações, imagens, sons, odores -, que são sugestões cognitivas internas ou ambientais pareadas ao uso da droga ${ }^{4}$. Esse fenômeno acontece com o uso repetido, quando os estímulos relacionados à droga passam a integrar uma rede associativa armazenada na memória 5 . Como consequência, os circuitos neurais podem se tornar hipersensíveis aos estímulos associados à substância ${ }^{6}$ e, quando o usuário é exposto a esses estímulos, os esquemas de ação são ativados, desencadeando um forte desejo, a necessidade de consumir a droga e o comportamento de procura por ela7. Em geral, a ativação do craving envolve multideterminantes ${ }^{8}$, podendo ser desencadeada tanto por estímulos internos - por exemplo, certas emoções/estados de humor - quanto externos (ambientais) - por exemplo, a presença de amigos, o local de uso, o toque de uma música -, que dependem do histórico de uso individual. Por isso, um dos principais objetivos do tratamento do dependente é descobrir quais são esses "gatilhos" e como eles podem desencadear uma situação de risco para a recaída.

Aparentemente, qualquer estímulo que foi repetidamente associado com o consumo de cocaína pode servir como gatilho para seu uso9. Pesquisas têm evidenciado que a nicotina possui um papel facilitador para o uso de cocaína e de outras drogas ilícitas ${ }^{10}$ e que pessoas tabagistas são mais propensas a usar cocaína e crack"11, assim como têm sugerido que a exposição à nicotina pode tanto aumentar a autoadministração de cocaína ${ }^{12}$ quanto servir como gatilho para disparar o craving por cocaína, especialmente em usuários de crack $^{13}$. Estudos clínicos demonstram que doses agudas de cocaína podem provocar um incremento na frequência de fumar ${ }^{14}$ assim como um aumento de grau de prazer e de paladar derivado dos cigarros ${ }^{13}$.
Por serem ambas estimulantes do SNC, a cocaína e a nicotina têm efeitos neuroquímicos interativos semelhantes e são capazes de estimular diretamente a via dopaminérgica ${ }^{15}$. No caso do crack (cocaína) e do tabaco (nicotina), por serem administradas pela via pulmonar, é provocada, quase imediatamente, uma inundação dopaminérgica ${ }^{16}$. Além disso, a nicotina e a cocaína têm efeitos subjetivos similares ${ }^{17}$ : ambas podem produzir efeitos adversos no humor e efeitos equivalentes no desempenho de tarefas e em medidas fisiológicas, tais como a frequência cardíaca e a pressão arterial18.

Embora esses achados da literatura não necessariamente proveem uma relação causal entre fumar tabaco e abusar de cocaína, eles demonstram um padrão convincente de associação entre ambas as drogas ${ }^{17}$.

Assim, o objetivo deste estudo foi verificar se há associação entre o aumento do craving pelo crack e o aumento do craving pelo tabaco em pacientes internados para desintoxicação.

\section{MÉTODO}

O projeto desta pesquisa foi primeiramente aprovado pelo Comitê de Ética em Pesquisa do Hospital Psiquiátrico São Pedro (HPSP/RS), e a assinatura do Termo de Consentimento Livre e Esclarecido (TCLE) foi considerada como pré-condição para a participação.

Este estudo caracteriza-se como um ensaio clínico do tipo quase-experimental. A amostra foi "por conveniência", composta por pacientes internados em uma Unidade de Desintoxicação Masculina, que tinham diagnóstico de dependência de cocaína (crack) e de tabaco pela CID-10 (Organização Mundial de Saúde, 1993). Eles tinham o crack como droga de escolha e tinham usado essa substância, pela última vez, há, no mínimo, duas e, no máximo, três semanas. Inicialmente, foram pesquisados 46 sujeitos, sendo excluídos 14, por estarem desmotivados para responder aos questionários, portarem diagnóstico de retardo mental e/ou apresentarem sintomas psicóticos. A amostra final foi de 32 sujeitos.

Os instrumentos utilizados foram:

- Inventário Beck de Ansiedade (BAI) - desenvolvido por Beck e Steer (1993), validado para o Brasil por Cunha $^{19}$. Consiste em um questionário que tem por objetivo medir a gravidade dos sintomas de ansiedade e é composto por 21 itens, com quatro opções de respostas $(0=$ absolutamente não, $1=$ levemente, 2 = moderadamente e 3 = gravemente). 0 escore total é obtido pelo somatório dos escores de cada item. Os pontos de corte para pacientes psiquiátricos são: de 0 a 10: mínimo; de 11 a 19: leve; de 20 a 30: moderado; e de 31 a 63: grave. Foi aplicado antes e depois da intervenção.

- Fagerström Test for Nicotine Dependence (FTND) - desenvolvido por Fagerström (1978), readaptado por 
Healtherton et al. (1991) e validado para tabagistas no Brasil por Carmo e Pueyo ${ }^{20}$. É um teste que avalia o padrão típico de fumar e classifica a dependência de nicotina em leve, moderada ou severa. Foi aplicado somente antes da intervenção.

- Cocaine Craving Questionnaire-Brief (CCQB) - desenvolvido por Sussner et al. (2006) e em processo de validação no Brasil por Araujo et al.21 para avaliar a presença do craving por cocaína. É composto por dez questões em uma escala analógica visual de sete pontos. Os participantes circulam a resposta de 1 (discordo totalmente) até 7 (concordo totalmente). A versão aplicada foi adaptada para o $\mathrm{crack}^{21}$ e a escala foi utilizada antes e depois da intervenção.

- Questionnaire of Smoking Urges-Brief (QSUB) - é uma escala abreviada elaborada por Cox et al. (2001) do Questionnaire of Smoking Urges (Tiffany e Drobes, 1991), validada no Brasil por Araujo et al. ${ }^{22}$ e utilizada para avaliar a presença do craving por tabaco. O QSUB é composto por dez questões afirmativas, diante das quais o indivíduo se posiciona utilizando uma escala de sete pontos que vai de "discordo totalmente" até "concordo totalmente". O QSUB pode ser analisado por meio do somatório total de pontos, dos pontos das categorias, dos pontos dos fatores 1 - questões 1, 3, 7 e 10 -, que avaliam o craving associado ao reforço positivo do uso do tabaco (por exemplo, "antecipação do efeito positivo"), e dos pontos dos fatores 2 - questões 4, 8 e 9 -, que avaliam o craving associado ao reforço negativo do tabaco (por exemplo, "alívio dos sintomas de abstinência ou afeto negativo"). Os pontos de corte para o total de pontos da escala são: de 0 a 13 pontos, craving mínimo; de 14 a 26, leve; de 27 a 42, moderado; e de 43 ou mais pontos, craving intenso. Foi aplicado antes e depois da intervenção.

- Ficha de Dados Sociodemográficos (FSD) e referentes ao padrão de consumo de substâncias psicoativas - questionário construído para identificar as características gerais da amostra e fatores relacionados ao consumo de crack e outras substâncias psicoativas. Foi aplicada somente após a intervenção.

Os dados foram coletados em nove intervenções realizadas em grupo, com duração de 40 minutos, e em entrevistas individuais, com duração de 25 minutos. $O$ grupo deveria ser constituído de, no mínimo, três e, no máximo, seis participantes, por adesão voluntária.

No dia da intervenção em grupo, todos os pacientes internados na UD que preenchiam os critérios de inclusão e que desejassem participar do estudo foram conduzidos a uma sala fechada com poucas interferências externas (luz, calor, ruídos), onde se sentaram em cadeiras dispostas em círculo em torno de uma mesa. Após, cada um dos participantes se apresentou dizendo seu nome e que drogas já havia experimentado. Inicialmente, foram avaliados individualmente os sintomas de ansiedade, o craving por tabaco e o craving por cocaína dos pacientes, por meio dos instrumentos BAI, CCQB e QSUB. Em um segundo momento, foi utilizado o método de exposição de imagens desenvolvido por Tifanny e Drobes $^{23}$, em que oito imagens fotográficas relativas ao crack e seu uso ("pedras", cachimbos, latas) foram apresentadas em folhas no tamanho A4, durante 1 minuto. Em seguida, foram reaplicados os instrumentos BAI, CCQB e QSUB, a fim de avaliar se houve indução do craving e dos sintomas de ansiedade. No intuito de delinear o perfil da amostra selecionada e avaliar se os sujeitos preenchiam mais algum critério de exclusão, foi realizada uma entrevista individual com aqueles participantes que concordaram em participar da intervenção até o fim, quando, então, se aplicou a FSD.

Os dados coletados foram analisados no programa Statistical Package for the Social Sciences - SPSS - versão 12.0. Os dados foram tratados estatisticamente, sendo feita uma Análise Descritiva e de Frequência dos dados sociodemográficos e referentes ao padrão de consumo de substâncias psicoativas. Foi utilizado o teste $t$ de Student para amostras pareadas para o estudo inferencial. O nível de significância foi de 5\%.

\section{RESULTADOS}

Quanto às características da amostra, a média de idade dos participantes foi de 24,63 anos (SD = 4,64; 18-40) e a média de anos de escolaridade foi de 8,25 anos (SD $=1,93$; 4-12). Quanto ao estado civil, 66,7\% eram solteiros, $12,1 \%$, casados e 18,2\%, separados/divorciados. Quanto à procedência, 9,1\% eram de Porto Alegre, 72,7\%, da grande Porto Alegre, 6,1\%, do interior do Rio Grande do Sul (RS) e 9,1\%, do litoral do RS. Quanto à ocupação, 12,2\% estavam desempregados, 30,3\% trabalhavam com carteira assinada, 42,4\% eram autônomos e 6,1\% estavam em licença-saúde, recebendo benefício do INSS. A renda mensal média foi de $\mathrm{R} \$ 606,56$ (SD = 313,04; 0-1200), aproximadamente 1,46 salário-mínimo (SM) utilizando-se como base de cálculo o SM de junho de 2008.

Com relação ao uso de substâncias psicoativas, 100\% eram dependentes de crack, 100\%, de tabaco, 57,6\%, de álcool, $93,9 \%$, de maconha e $24,2 \%$, de solventes. A idade de início do uso de tabaco foi, em média, 14 anos (SD = 4,095; 9-31) e de início do uso de crack, em média, de 19,50 anos $(S D=4,586 ; 14-34)$. Quanto ao uso do crack, os pacientes consumiam uma média de 67,73 pedras por semana (SD $=62,37 ; 14-280$ ). Quanto à gravidade do tabagismo medida pela Fagerström, 14 eram leves (43,8\%), 11, moderados $(34,4 \%)$ e 7 , graves $(21,9 \%)$.

Os resultados deste estudo demonstraram, segundo autorrelato dos pacientes, que o craving por crack pode ser desencadeado por vários gatilhos: usar álcool (em 81,3\% da amostra; $n=26$ ), sentir emoções (raiva, tristeza, alegria) 
(96,9\%; $n=31)$, ter dinheiro na mão $(90,6 \% ; n=29)$, ver usuários de $\operatorname{crack}(78,1 \% ; n=25)$, sentir-se sozinho $(40,6 \% ; n=13)$ e outros $(20,1 \% ; n=9)$.

Na tabela 1, é apresentada a comparação, por meio do teste t de Student para amostras pareadas, das médias e desvios-padrão de craving e ansiedade antes e depois da apresentação das imagens do crack e, na tabela 2, são apresentadas, segundo o coeficiente de correlação linear de Spearman, as correlações relacionadas às pontuações no craving.

Tabela 1. Comparação das médias e desvios-padrão de craving e ansiedade antes e depois da apresentação das imagens do crack $(n=32)$

\begin{tabular}{lllllc}
\hline & Variáveis & M & SD & T & P \\
\hline CCQB & CCQB antes & 19,03 & 5,43 & & \\
& CCQB depois & 25,06 & 10,86 & $-3,71$ & 0,001 \\
QSUB & QSUB antes & 32,03 & 19,30 & & \\
& QSUB depois & 38,91 & 20,12 & $-4,981$ & $<0,001$ \\
QSUB & QSUB Fator 1 antes & 15,53 & 8,59 & & \\
Fator 1 & QSUB Fator 1 depois & 18,41 & 8,87 & $-4,41$ & $<0,001$ \\
\multirow{2}{*}{ QSUB } & QSUB Fator 2 antes & 7,72 & 6,08 & & \\
Fator 2 & QSUB Fator 2 depois & 9,34 & 6,41 & $-3,14$ & 0,004 \\
BAI & BAl antes & 6,75 & 5,41 & & \\
& BAl depois & 12,84 & 7,76 & $-5,85$ & $<0,001$
\end{tabular}

CCQB total antes: pontuação no Cocaine Craving Questionnaire-Brief (craving por crack) antes da intervenção; CCQB total depois: pontuação no Cocaine Craving Questionnaire-Brief (craving por crack) depois da intervenção; QSUB tota antes: pontuação no Questionnaire Smoking Urges-Brief (craving por tabaco) antes da intervenção;

QSUB fator 1 antes: pontuação no fator 1 (reforço positivo) do Questionnaire Smoking Urges-Brief (craving por tabaco) antes da intervenção;

QSUB fator 2 antes: pontuação no fator 2 (reforço negativo) do Questionnaire Smoking Urges-Brief (craving por tabaco) antes da intervenção;

QSUB total depois: pontuação no Questionnaire Smoking Urges-Brief (craving por tabaco) depois da intervenção;

QSUB fator 1 depois: pontuação no fator 1 (reforço positivo) do Questionnaire Smoking Urges-Brief (craving por tabaco) depois da intervenção;

QSUB fator 2 depois: pontuação no fator 2 (reforço negativo) do Questionnaire Smoking Urges-Brief (craving por tabaco) depois da intervenção;

BAl antes; pontuação no Inventário Beck de Ansiedade (sintomas de ansiedade) antes da intervenção;

BAI Depois: pontuação no Inventário Beck de Ansiedade (sintomas de ansiedade) depois da intervenção.

\section{DISCUSSÃO}

Os achados da literatura relatam que os dependentes de cocaína, em geral, abusam de múltiplas drogas ou têm histórico de consumo de outras substâncias psicoativas ${ }^{24,25}$. Concordando, em nossa amostra, os dependentes de crack (100\%) são também dependentes de tabaco (100\%), de maconha (93,9\%), de álcool $(57,6 \%)$ e de solventes (24,2\%). Tal uso múltiplo de drogas pode produzir efeitos clínicos e comportamentais adversos, cumulativos e sinérgicos, via interação entre as substâncias, podendo afetar o resultado do tratamento para dependência de cocaína ${ }^{17}$.

Para a maioria dos indivíduos desta amostra, segundo autorrelato, o craving por crack pode ser desencadeado por estímulos externos - usar álcool (81,3\%), ter dinheiro na mão (90,6\%), ver usuários de crack $(78,1 \%)$ - e por estímulos in-
Tabela 2. Correlações relacionadas às pontuações no craving do crack e do tabaco

\begin{tabular}{|c|c|c|c|c|c|c|c|c|}
\hline & $\begin{array}{l}\text { CCQB } \\
\text { Total } \\
\text { antes }\end{array}$ & $\begin{array}{l}\text { CCQB } \\
\text { Total } \\
\text { depois }\end{array}$ & $\begin{array}{l}\text { QSUB } \\
\text { Total } \\
\text { antes }\end{array}$ & $\begin{array}{l}\text { QSUB } \\
\text { Fator } 1 \\
\text { antes }\end{array}$ & $\begin{array}{l}\text { QSUB } \\
\text { Fator } 2 \\
\text { antes }\end{array}$ & $\begin{array}{l}\text { QSUB } \\
\text { Total } \\
\text { depois }\end{array}$ & $\begin{array}{l}\text { QSUB } \\
\text { Fator } 1 \\
\text { depois }\end{array}$ & $\begin{array}{l}\text { QSUB } \\
\text { Fator } 2 \\
\text { depois }\end{array}$ \\
\hline $\begin{array}{l}\text { CCQB } \\
\text { Total } \\
\text { antes }\end{array}$ & 1,000 & $0,683^{* *}$ & 0,335 & 0,258 & $0,389^{*}$ & $0,406^{*}$ & 0,307 & $0,576^{* *}$ \\
\hline $\begin{array}{l}\text { CCQB } \\
\text { Total } \\
\text { depois }\end{array}$ & $0,683^{* *}$ & 1,000 & 0,301 & 0,228 & 0,334 & $0,442^{*}$ & $0,351^{*}$ & $0,524^{* *}$ \\
\hline $\begin{array}{l}\text { QSUB } \\
\text { Total } \\
\text { antes }\end{array}$ & 0,335 & 0,301 & 1,000 & $0,939^{* *}$ & $0,858^{* *}$ & $0,924^{* *}$ & $0,859^{* *}$ & $0,676^{* *}$ \\
\hline $\begin{array}{l}\text { QSUB } \\
\text { Fator } 1 \\
\text { antes }\end{array}$ & 0,258 & 0,228 & $0,939^{* *}$ & 1,000 & $0,659 * *$ & $0,881^{* *}$ & $0,912^{* *}$ & $0,531^{* *}$ \\
\hline $\begin{array}{l}\text { QSUB } \\
\text { Fator } 2 \\
\text { antes }\end{array}$ & $0,389^{*}$ & 0,334 & $0,858^{* *}$ & $0,659 * *$ & 1,000 & $0,788^{* *}$ & $0,593^{* *}$ & $0,805^{* *}$ \\
\hline $\begin{array}{l}\text { QSUB } \\
\text { Total } \\
\text { depois }\end{array}$ & $0,406^{*}$ & $0,442^{*}$ & $0,924^{* *}$ & $0,881^{* *}$ & $0,788^{* *}$ & 1,000 & $0,884^{* *}$ & $0,810^{* *}$ \\
\hline $\begin{array}{l}\text { QSUB } \\
\text { Fator } 1 \\
\text { depois }\end{array}$ & 0,307 & $0,351^{*}$ & $0,859 * *$ & $0,912^{* *}$ & $0,593^{* *}$ & $0,884^{* *}$ & 1,000 & $0,560^{* *}$ \\
\hline $\begin{array}{l}\text { QSUB } \\
\text { Fator } 2 \\
\text { depois }\end{array}$ & $0,576^{* *}$ & $0,524^{* *}$ & $0,676^{* *}$ & $0,531^{* *}$ & $0,805^{* *}$ & $0,810^{* *}$ & $0,560^{* *}$ & 1,000 \\
\hline $\begin{array}{l}\text { BAl } \\
\text { antes }\end{array}$ & $0,394^{*}$ & $0,391^{*}$ & $0,439^{*}$ & 0,254 & $0,503^{* *}$ & $0,376^{*}$ & 0,266 & $0,401^{*}$ \\
\hline $\begin{array}{l}\text { BAI } \\
\text { depois }\end{array}$ & $0,483^{* *}$ & $0,684^{* *}$ & 0,268 & 0,103 & $0,418^{*}$ & $0,401^{*}$ & 0,284 & $0,557^{* *}$ \\
\hline FTND & 0,248 & $0,390^{*}$ & $0,391^{*}$ & 0,338 & $0,362^{*}$ & $0,404^{*}$ & 0,349 & 0,346 \\
\hline
\end{tabular}

ternos como sentir emoções - raiva, tristeza, alegria $(96,9 \%)$. Esses resultados sugerem algumas situações de risco de recaída importantes de serem avaliadas no tratamento desses indivíduos ${ }^{1}$

As pesquisas demonstram que a exposição do indivíduo a "gatilhos" ambientais previamente associados com o uso de cocaína produz uma resposta fortemente condicionada, caracterizada pela hiperexcitação autonômica e pelo aumento nas medidas subjetivas do craving por cocaína ${ }^{26,27}$. A partir da exposição de imagens relativas ao uso de crack neste estudo, houve significativa indução do craving pelo crack. No entanto, alguns pacientes relataram que o "gatilho" que induziu o craving pelo crack não foram as imagens fotográficas, mas sim as falas e o comportamento dos outros pacientes, que se alteraram no momento da apresentação das imagens, no qual gírias e trejeitos peculiares e característicos do uso de crack foram observados pelo pesquisador. Portanto, essa ocorrência da comunicação verbal e não verbal entre os participantes durante a intervenção não nos permite atribuir o aumento do craving somente à exposição das imagens. 
Somente uma minoria negou a indução do craving pelas imagens, fato que pode ter ocorrido pela dificuldade de reconhecer em si o craving, pelo medo de relatar o craving e ficar mais tempo internado - mesmo com a explicação do TCLE - ou por estarem em ambiente protegido, continente e afastado do acesso à droga.

Foi encontrada uma associação significativa entre o aumento do craving pelo crack e o aumento do craving pelo tabaco em pacientes internados para desintoxicação, propondo, assim, a existência de uma relação estreita entre o uso de crack e o uso de tabaco ${ }^{10-14}$. Um dado interessante foi que o craving pelo crack antes da intervenção teve correlação positiva de intensidade moderada somente com o total de pontos do QSUB e com o fator 2 do QSUB, responsável pelo craving por causa do alívio dos sintomas de abstinência e do afeto negativo, enquanto, depois da intervenção, ao ser confrontado com o estímulo, foram observadas correlações positivas de intensidades moderadas com o total de pontos do QSUB e tanto com o fator 2 quanto com o fator 1, o qual é responsável pela antecipação do efeito positivo do tabaco. Esse fenômeno demonstra o que Beck et al. ${ }^{1}$ descrevem como a ação de crenças antecipatórias quanto ao efeito positivo da substância que se ativam juntamente com o craving quando o indivíduo tem contato com situações-estímulos relacionadas ao uso da droga.

Entre o crack (cocaína) e o tabaco, a semelhança na ação neuroquímica - rápida e intensa - e a similaridade dos efeitos subjetivos estimulantes ${ }^{15-18}$ podem explicar por que essas substâncias são usadas de modo concorrente com grande frequência e por que ocorreu, em nosso estudo, uma correlação positiva consistente entre o craving por crack e o craving por tabaco, tanto antes como depois da intervenção.

Outra explicação possível para esse resultado é de caráter comportamental. Além da semelhança biomecânica ${ }^{13}$ para usar a "pedra", conforme relato dos pacientes, é preciso colocar cinzas de cigarro na base do cachimbo ou lata para que haja sua combustão. Ou seja, adicionalmente à similaridade do ato motor de fumar, necessita-se obrigatoriamente do uso do tabaco para consumir crack. Em nossa amostra, alguns dependentes de crack mencionaram que iniciaram o consumo do tabaco a partir do uso do crack, por causa de sua forma de administração. Aqueles que relataram o consumo do tabaco antes do início de uso do crack referiram aumento na frequência de fumar tabaco e na quantidade de cigarros a partir do início do uso do crack, principalmente nos períodos de uso compulsivo deste último. Esses comportamentos modificados (início e aumento do tabagismo) também podem estar ligados à correlação positiva entre o craving por crack e o craving por tabaco encontrada nesta pesquisa, pois a memória do uso do crack pode ter servido como "gatilho" para o consumo do tabaco. Embora já tenha sido citada na literatura ${ }^{28}$, essa hipótese comportamental deve ser investigada mais criteriosamente em estudos posteriores.
Os resultados do nosso estudo demonstraram correlações positivas de intensidade moderada entre os sintomas de ansiedade e o craving por crack antes da intervenção e o craving por tabaco, tanto antes como depois da intervenção, assim como correlação positiva de intensidade alta entre os sintomas de ansiedade e craving por crack depois da intervenção. Esses achados propõem a existência de mecanismos comuns subjacentes a ambos, o que nos leva a deduzir que o aumento dos sintomas de ansiedade está relacionado com o aumento do craving e vice-versa ${ }^{29}$. No entanto, como os pacientes não foram avaliados neste estudo quanto à existência de comorbidades psiquiátricas, altamente prevalentes em dependentes de drogas, que podem produzir aumento de sintomas depressivos/ansiosos, não se sabe se o aumento dos sintomas de ansiedade está relacionado somente ao aumento do craving. De qualquer forma, independentemente da existência ou não de comorbidades psiquiátricas, sugere-se que a aprendizagem do manejo dos sintomas de ansiedade, por fazerem parte da sintomatologia do craving, deva estar incluída no tratamento para dependência de crack a fim de evitar uma recaída.

É importante destacar que a alta prevalência de dependência de maconha em nossa amostra pode ser considerada um fator confundidor que pode ter afetado os resultados encontrados neste estudo, já que essa substância também é utilizada pela via fumada apresentada nos estímulos visuais.

\section{CONCLUSÕES}

Em virtude da ocorrência de uma relação estreita entre o uso de cocaína e o uso do tabaco ${ }^{10-14}$ e das evidências de que a exposição à nicotina pode tanto aumentar a autoadministração de cocaína ${ }^{12}$ quanto servir como "gatilho" para disparar o craving por cocaína, especialmente em usuários de crack $^{13}$, pode-se afirmar que foi demonstrada, neste estudo, a existência de associação entre o craving por crack e o craving por tabaco. Como uma estratégia bem-sucedida para o tratamento da dependência de psicoestimulantes, deve-se incluir o tratamento para dependência de nicotina, pois, por causa do papel proeminente dos receptores nicotínicos nos circuitos de recompensa de drogas ${ }^{30}$, o tratamento sinérgico das duas dependências (crack e tabaco) nesses pacientes aumentaria as chances de evitar uma recaída para uso do crack. Portanto, sugere-se que, para a eficácia do tratamento para dependência de crack, o usuário também deve ser motivado para a abstinência do tabaco.

\section{CONFLITOS DE INTERESSES}

Este estudo não apresenta conflitos de interesse. 


\section{REFERÊNCIAS}

1. Beck A, Newman C, Wright F. Terapia cognitiva de las drogodependencias. Barcelona: Ediciones Paidos Ibérica; 2000.

2. Araujo RB, Oliveira MS, Pedroso RS, Miguel AC, Castro MGT. Craving e dependência química: conceito, avaliação e tratamento. J Bras Psiquiatr. 2008:57(1):57-63.

3. Nappo SA, Galduróz JC, Raymundo M, Carlini EA. Changes in cocaine use as viewed by key informants: a qualitative study carried out in 1994 and 1999 in São Paulo, Brazil. J Psychoactive Drugs. 2001;33(3):241-53.

4. Edwards G, Dare C. Psicoterapia e tratamento das adições. Porto Alegre: Artes Médicas; 1997. p. 247

5. Johnsen HB, Laberg CJ, Cox MW, Vaksdal A, Hugdahl K. Alcoholic subjects' attentional bias in the processing of alcohol-related words. Psychol Addict Behav. 1994;8:111-5.

6. Robinson TE, Berridge KC. The neural basis of drug craving: an incentive-sensitization theory of addiction. Brain Res Rev. 1993;18:247-91.

7. Conklin AC, Tiffany ST. Applying extinction research and theory to cue-exposure addiction treatments. Addiction. 2002;97:155-67.

8. Wallace BC. Psychological and environmental determinants of relapse in crack cocaine smokers. J Subst Abuse Treat. 1989;6(2):95-106.

9. Siegel RK. Cocaine smoking disorders: diagnosis and treatment. Psychiatr Ann. 1984;14(10):728-32.

10. Martinez-Ortega JM, Jurado D, Martinez-Gonzalez MA, Gurpegui M. Nicotine dependence, use of illegal drugs and psychiatric morbidity. Addict Behav. 2006;31(9):1722-9.

11. Lai S, Lail H, Page JB, McCoy CB. The association between cigarette smoking and drug abuse in the United States. J Addict Dis. 2000;19(4):11-24.

12. Horger BA, Giles MK, Schenk S. Pre-exposure to amphetamine and nicotine predisposes rats to self-administer a low dose of cocaine. Psychopharmacology. 1992;107:271-6. Disponivel em: http://www.ncbi.nlm.nih.gov/entrez/utils/fref.fcgi?Prld=3051\&itool=Abstr actPlus-def\&uid=9862770\&db=pubmed\&url=http://jpet.aspetjournals.org/cgi/pmidlo okup?view=long\&pmid=9862770. Acesso em: 4 dez 2009.

13. Sees KL, Clark HW. When to begin smoking cessation in substance abusers. J Subst Abuse Treat. 1993;10:189-95.

14. Roll JM, Higgins ST, Tidey JW. Cocaine use can increase cigarette smoking: evidence from laboratory and naturalistic settings. Exp Clin Psychopharmacol. 1997;5(3):263-8.
15. Di Chiara G. The role of dopamine in drug abuse viewed from the perspective of its role in motivation. Drug Alcohol Depend. 1995;38:95-137.

16. Pontiere FE, Tanda G, Di Chiara G. Intravenous cocaine, morphine, and amphetamine preferentially increase extracellular dopamine in the "shell" as compared with "core" of the rat nucleus accumbens. Proc Natl Acad Sci U S A. 1996;92:12304-8.

17. Reid MS, Mickallan JD, Delucchi KL, Hall SM, Berger SP. An acute dose of nicotine enhances cue-induced cocaine craving. Drug Alcohol Depend. 1998;49(2):95-104.

18. Higgins ST, Bickel WK, Hughes JR, Lynn M, Capeless MA. Effects of intranasal cocaine on human learning, performance and physiology. Psychopharmacology. 1990;102(4):451-8.

19. Cunha JA. Manual da versão em português das Escalas Beck. São Paulo: Casa do Psicólogo; 2001, $171 \mathrm{p}$.

20. Carmo JT, Pueyo A. A adaptação do português do Fagerström Test for Nicotine Dependence (FTND) para avaliar a dependência e tolerância à nicotina em fumantes brasileiros. Rev Bras Med. 2002;59(12):73-80.

21. Araujo RB, Pedroso RS, Castro MGT. A adaptação transcultural para o idioma português do Cocaine Craving Questionnaire Brief. Rev Psiquiatr Clin. 2010;37(5):195-8.

22. Araujo RB, Oliveira MS, Moraes JFD, Pedroso RS, Port F, Castro MGT. Validação da versão brasileira do Questionnaire of Smoking Urges-Brief. Rev Psiquiatr Clin. 2007;34(4):166-75. Disponível em: http://www.scielo.br/scielo.php?script=sci_arttext \&pid=\$0101608320 07000400002\&lng=en\&nrm=iso. Acesso em: 4 dez 2009.

23. Tiffany ST, Drobes DJ. Imagery and smoking urges: the manipulation of affective content. Addict Behav. 1990;15(6):531-9.

24. Ferreira Filho 0. Perfil sociodemográfico e de padrões de uso entre dependentes de cocaína hospitalizados. Rev Saude Publica. 2003;37(6):751-9.

25. Duailibi LB, Ribeiro M, Laranjeira R. Perfil dos usuários de cocaína e crack no Brasil. Cad Saude Publica. 2008;24(4):545-57.

26. Ehrman RN, Robbins SJ, Childress AR, O'Brien CP. Conditioned responses to cocaine-related stimuli in cocaine abuse patients. Psychopharmacology. 1992;107:523-9.

27. $O^{\prime}$ Brien CP, Childress AR, McLellan T, Ehrman R. Integrating systemic cue exposure with standard treatment in recovering drug dependent patients. Addict Behav. 1990;15:355-65.

28. Wiseman EJ. Nicotine dependence and withdrawal in cocaine-dependent patients. Arch Gen Psychiatry. 2000;56:85-9.

29. Roelofs S. Hyperventilation, anxiety, craving for alcohol: a subacute alcohol withdrawal syndrome. Alcohol. 1985;2(3):501-5.

30. Bechtholt A, Mark G. Enhancement of cocaine-seeking behavior by repeated nicotine exposure in rats. Psychopharmacology (Berl). 2002;162(2):178-85. 